\title{
TMS-induced theta phase synchrony reveals a bottom-up network in working memory
}

\section{Short title:}

Bottom-up network in working memory

\section{Authors:}

Eri Miyauchi ${ }^{1}$, Keiichi Kitajo ${ }^{2,3}$, and Masahiro Kawasaki ${ }^{1,2,3}$

1. Department of Intelligent Interaction Technology, Graduate School of Systems and Information Engineering, University of Tsukuba, Japan

2. Rhythm-based Brain Computation Unit, RIKEN BSI-TOYOTA Collaboration Center, Japan

3. Laboratory for Advanced Brain Signal Processing, RIKEN Brain Science Institute, Japan

\section{Address correspondence to:}

Dr. Masahiro Kawasaki

University of Tsukuba

1-1-1, Tennodai, Tsukuba-shi, Ibaraki 305-8573, Japan

kawasaki@iit.tsukuba.ac.jp 
Conflicts of interest: none declared

Source of funding: Grant-in-Aid for Scientific Research on Innovative areas (21120005 and 25119512), Grant-in-Aid for Scientific Research (B) (26282169), JST PRESTO, and Program to Disseminate Tenure Tracking System MEXT.

Manuscript information:

Number of Character of text: 13,978 


\section{Abstracts}

Global theta phase synchronization between the frontal and sensory areas has been suggested to connect the relevant areas for executive processes of working memory (WM). However, little is known regarding network directionality (i.e. top-down or bottom-up) of this interaction. To address the issue, the present study conducted transcranial magnetic stimulation (TMS) - electroencephalography (EEG) experiment during WM tasks. Results showed that TMS-induced increases in theta phase synchronization were observed only when TMS was delivered to the sensory areas but not the frontal area. These findings suggest that network directionality represented in WM is bottom-up rather than top-down.

\section{Keywords}

Theta, synchronization, TMS, working memory, EEG 


\section{Introduction}

Neural substrates of working memory (WM) are thought to be separate systems; the prefrontal area for the executive system and posterior sensory areas for the maintenance system, including parietal areas for visual WM and temporal areas for auditory WM [1-3]. Recent human electroencephalography (EEG) studies have shown that a global network with large-scale phase synchronization has an important role in WM [4-6]. In particular, theta rhythms in distributed brain regions are considered to interact with each other $[7,8]$. Moreover, it has been suggested that low-frequency synchronizations connect the frontal area with posterior sensory areas for the executive system $[9,10,11]$. However, network directionality of such interactions in WM is not clear; either top-down or bottom-up mechanisms which are defined as signals from sensory to frontal areas or signals from frontal to sensory areas, respectively in this study. Previous studies based on transcranial magnetic stimulation (TMS) and EEG have suggested that single-pulse TMS can manipulate local synchronization in-targeted neural areas [12-14] and induce spatial propagation during a resting state [15]. It is plausible that this method could identify network directionality among WM-relevant brain regions by focusing on TMS-induced changes in EEG rhythms during WM tasks. For example, if phase synchronization changes when TMS is 
delivered to the frontal cortex, directionality is likely to be top-down. In contrast, if phase synchronization changes when TMS is delivered to the sensory cortices, directionality is likely to be bottom-up.

Hence, this study aim to clarify WM network directionality. Two types of WM manipulation tasks [an auditory WM task (AWM) and a visual WM task (VWM)] were performed [10], and single-pulse TMS was delivered to three target areas (frontal area, visual area, auditory area). The tasks were also performed during sham-TMS and within a no-TMS condition.

\section{Methods}

\section{Participants}

Ten healthy right-handed volunteers (four females; mean age $=23.5 \pm 1.1$ years, range 20-33 years) with normal or corrected-to-normal visual acuity, normal hearing acuity, and normal motor performance took part in this EEG experiment. All participants gave written informed consent, and the protocol was approved by the Ethical Committee of the RIKEN (in accordance with the Declaration of Helsinki), before the experiments were performed.

\section{Auditory working memory task}


Participants, wearing earphones, faced a computer screen placed $60 \mathrm{~cm}$ away. At the beginning of each trial, participants were required to memorize a 1-digit number presented as an auditory stimulus through the earphones for $1 \mathrm{~s}$ (sample display, Figure 1a). After a 2-s retention interval, another 1-digit number was presented as an auditory stimulus for $1 \mathrm{~s}$, and participants were asked to add the presented number to the earlier memorized number. This mental addition (“manipulation phase”) was repeated 3 times, and a probe number was presented as an auditory stimulus, after a white fixation point had turned gray (test display). Participants had to determine whether the probe number matched the mental calculation total within $2 \mathrm{~s}$ (while the fixation point was red) by pressing a button. An inter-trial interval (ITI) duration was set at 2 s. Stimuli were generated using Matlab2010 with the Psychophysics Toolbox extension.

\section{Visual working memory task}

At the beginning of each trial, $5 \times 5$ square grids $\left(5^{\circ} \times 5^{\circ}\right)$ and a red circle $\left(1^{\circ} \times 1^{\circ}\right)$ were presented on the computer screen for $1 \mathrm{~s}$ (sample display, Figure 1b). Participants were required to memorize the position of the red circle. After a 2-s retention interval, participants needed to mentally move the red circle in accordance with a white arrow presented at the center of the screen for $1 \mathrm{~s}$ ("manipulation phase”). The arrow was directed upward, downward, rightward, or leftward. Participants were asked to repeat 
this mental manipulation 3 times and then indicate whether the mentally determined position of the red circle matched a visual probe (test display). Button presses, ITI duration, and stimulus creation were identical to the AWM task.

\section{TMS}

On each trial, three pulses of single-pulse TMS were applied to frontal (Fz), temporal (TP7), or parietal $(\mathrm{Pz})$ areas during the manipulation phase of the task. Specifically, for each manipulation cue (a number with a note symbol in Figure 1a for the AWM tasks or a white arrow in Figure 1b for the VMM tasks), a single pulse of TMS was applied at one of three cue-TMS stimulus onset asynchronies (0,500, and $1000 \mathrm{~ms})$. We used a figure-eight coil, with a 70-mm wing diameter connected to a biphasic stimulator (Magstim Rapid, Magstim Company Ltd., UK) for TMS application. For maintaining the coil position and direction throughout each session, the flexible arm of a camera stand was used. Prior to performing the experiments, the TMS intensity of each participant was determined as the 95\% motor threshold which was minimum intensity to make his/her index fingertwitch. To test placebo effects of TMS, the sham-TMS condition was conducted by applying TMS pulses to $15 \mathrm{~cm}$ from the top of the head.

\section{Experimental procedure}

Each participant completed 10 separate sessions; 2 WM tasks (AWM and VWM 
tasks) X 5 TMS conditions (frontal, temporal, parietal, sham, and no-TMS) in a counterbalanced order. Each session consisted of 24 trials (72 TMS applications). All participants were well trained before the EEG-measurement sessions.

\section{EEG recordings}

EEG recordings were performed using 67 scalp electrodes (Ag/AgCl), embedded in a TMS-compatible electrode cap (EasyCap; EASYCAP Gmbh, Germany), and in accordance with placement based on the international 10/10 system. The sampling rate was 1,000 Hz. Reference electrodes were located on the right and left mastoids. Electrode impedance was kept below $10 \mathrm{k} \Omega$. Electrooculography (EOG) was recorded from electrodes placed vertical and horizontal from the right and left eyes. The EEG and EOG signals were amplified using a BrainAmp MR+ apparatus (Brain Products, Germany).

\section{EEG data pre-processing}

We only analyzed EEG data for correct trials. EEG data was segmented to 3-s epochs for the manipulation period from the instruction onset for manipulation. We removed EEG data points affected by TMS artifacts (from -1 to $7 \mathrm{~ms}$ post-TMS onset) using linear interpolation [16]. The EEG epochs were subjected to infomax independent components analysis (ICA). ICA components that were significantly correlated with the 
vertical or horizontal EOGs were eliminated as ocular artifacts. ICA-corrected data were recalculated using regression for the remaining components [9]. To eliminate volume conduction errors, we performed current source density analysis at each electrode position $[17,18]$ and applied the spherical Laplace operator to the voltage distribution on the scalp surface.

\section{Wavelet analysis}

We applied wavelet transforms using Morlet's wavelet function [19]. Six time points were selected for the analysis $(0,500,1000,1500,2000$, and $2500 \mathrm{~ms})$. The phase for each time point at each TMS application was the arctangent of the original, convoluted EEG signal $s(t)$ results with a complex Morlet's wavelet function $w(t, f)$ :

$$
w(t, f)=\left(\sigma_{t} \sqrt{\pi}\right)^{-1 / 2} \exp \left(-t^{2} / 2 \sigma_{t}^{2}\right) \exp (i 2 \pi f t)
$$

where $\sigma_{t}$ is the standard deviation of the Gaussian window. The wavelet used was characterized by a constant ratio ( $\left.\mathrm{f} / \sigma_{\mathrm{f}}=7\right)$, with $f$ ranging from 2 to $20 \mathrm{~Hz}$ (1-Hz steps).

To identify the phase relations between any 2 electrodes, we calculated phase locking values (PLV) at time point (t) and frequency (f) as follows:

$$
\operatorname{PLV}(\mathrm{t})=\frac{1}{N}\left|\sum_{n=1}^{N} e^{i \Delta \theta_{j k}(t, f, n)}\right|
$$

where $\Delta \theta_{\mathrm{jk}}(t, f, n)$ is the phase difference between the $j$ th and $k$ th electrodes and the number of trials $N$ [20]. We first calculated the PLV for each subject and then compared 
the PLV on each time point for manipulation periods with the averaged PLV for baseline periods (i.e. ITI) by using the Wilcoxon sign rank test with Bonferroni correction. We made region-of-interest (ROI) analysis and selected $\mathrm{Fz}, \mathrm{TP7}$, and $\mathrm{Pz}$ as the representative frontal, temporal, and parietal electrodes in reference to our previous studies [9]. We evaluated the PLV between these three ROI electrodes and the other electrodes.

\section{Results}

\section{Behavioral results}

Subject-averaged accuracy rates ( \pm s.d.) during the AWM were as follows: $96.7 \pm 1.3$, $96.0 \pm 0.8,97.2 \pm 0.6,96.3 \pm 1.0$, and $96.5 \pm 1.2 \%$ for no, frontal, temporal, parietal, and sham-TMS conditions, respectively. Subject-averaged accuracy rates ( \pm s.d.) during the VWM were as follows: $96.9 \pm 1.3,95.6 \pm 1.3,96.0 \pm 1.1$, $96.3 \pm 1.5$, and $95.6 \pm$ $0.9 \%$ for no, frontal, temporal, parietal, and sham-TMS conditions, respectively. A 2-factor ANOVA revealed no main effect of task $\left(F_{1,90}=0.42, p=0.52\right)$, TMS conditions $\left(F_{4,90}=0.26, p=0.90\right)$, and no significant interaction $\left(F_{4,90}=0.14, p=0.97\right)$. These results indicated that EEG comparisons among the different conditions were not influenced by either task difficulty or TMS effects. 


\section{EEG results}

We identified electrode pairs showing the PLV at each time point was significantly higher than the averaged PLV for the baseline period ( $p<0.05$; Bonferroni correction). Since the present study investigated theta synchronization modulation, we focused on the theta-range (e.g. 4Hz) PLV between the frontal and other electrodes, between the temporal and other electrodes, and between the parietal and other electrodes. Figure 2 shows the significant pairs between the ROI electrodes and the other electrodes at each time point during the no-TMS, frontal-TMS and temporal-TMS (parietal-TMS) conditions during the AWM (VWM) tasks. Figure 2 showed the 1000ms-TMS results as the representative results. Figure 3 shows the average counted number of electrode pairs showing significantly higher theta $(4 \mathrm{~Hz})$ PLV for manipulation periods than that for ITI periods ( $p<0.05$; Bonferroni correction) among the 6 latencies (0ms, 500ms, 1000ms, 1500ms, 2000ms, and 2500ms) and among the 3 TMS application timings (0ms, 500ms, and 1000ms). The no-TMS condition included several significant pairs: between the frontal and other electrodes and between the temporal (parietal) and other electrodes during the AWM (VWM) tasks. These results were similar to those from the frontal-TMS and sham-TMS conditions. Interestingly, sensory-TMS (i.e. temporal-TMS and parietal-TMS) conditions significantly increased the number of significant pairs 
between the frontal and other electrodes and between the TMS-targeted and other electrodes in comparison with the no-TMS, frontal-TMS, and sham-TMS conditions ( $p$ $<$ 0.05; Chi-squared test with Bonferroni corrected multiple comparison). These tendencies (i.e the number of significant pairs) were almost the same among TMS application timing.

The above results for the analyses using the single time point of EEG data might be sensitive to noise or extreme points. Therefore, the analyses need to be redone averaging over longer windows than a single time point. We averaged the PLV data over 100 msec time windows; -50 msec to 50 msec from the onset of the TMS application and conducted the same statistical analyses under all the conditions. As the results, the number of electrodes showing significant connectivity was 0 (from the frontal electrode) and 0 (from the temporal electrode) under no-TMS, 0 (from the frontal electrode) and 0 (from the temporal electrode) under frontal-TMS, and 7 (from the frontal electrode) and 8 (from the temporal electrode) under temporal-TMS, during auditory WM conditions; 3 (from the frontal electrode) and 3 (from the parietal electrode) under no-TMS, 4 (from the frontal electrode) and 3 (from the parietal electrode) under frontal-TMS, and 7 (from the frontal electrode) and 8 (from the parietal electrode) under temporal-TMS, during visual WM conditions. These results were 
almost same as the results for the analysis using the single time point data.

\section{Discussion}

The present study elucidated a bottom-up network in WM through functional changes in theta phase synchronization induced by TMS. Consistent with earlier studies suggesting that theta phase synchronization reflects a global connection among relevant brain regions [5, 8, 9], theta phase synchronization was observed between WM task-relevant areas: between the frontal and parietal areas during a VWM task and between the frontal and temporal areas during an AWM task. As expected from a previous study, which suggested that single-pulse TMS modulates global phase synchronization and information flow among brain networks at rest [15], TMS manipulated brain activity with global theta phase synchronization during WM tasks. Our EEG data revealed a significant difference in the amount of TMS-induced changes in theta phase synchronization between TMS-targeted areas. Additionally, TMS-induced changes in theta phase synchronization indicated that network directionality was bottom-up rather than top-down. In the parietal-TMS condition during the VWM task, induced theta phase synchronization from both frontal and parietal areas increased. In the temporal-TMS condition during the AWM task, induced theta phase synchronization 
from both frontal and temporal areas increased. Although there were slight changes in theta phase synchronization in the parietal-TMS condition during the AWM task and in the temporal-TMS condition during the VWM task, these results are modality specific; therefore, it is possible that network directionality during the WM tasks was bottom-up. Note that in the frontal-TMS condition, results from both the VWM and AWM tasks were similar to the no-TMS condition. These results show that there was no increase in induced theta phase synchronization in the frontal-TMS condition; thus, these results indicate that network directionality during the WM tasks was not top-down. It should be noted results in the sham-TMS condition were similar to the no-TMS condition. These results suggest that our findings were not influenced by auditory evoked responses induced by the associated TMS "clicking" sound during the experiment.

Induced theta phase synchronization was increased only when TMS was delivered to the sensory areas, not the frontal areas. Thus, it could be argued the information network employed during the WM tasks were bottom-up rather than top-down. This proposal is supported by previous findings regarding the effects of TMS on EEG signals. Previous studies have shown that TMS manipulates brain activation not only in TMS-targeted areas [12-14] but also in relevant non-TMS targeted areas [21-23]. Furthermore, a single-pulse TMS to sensory areas, but not motor areas, increases theta phase 
synchronization among sensory and motor areas during a resting state. This reflects processing in the default mode network. The information theory (i.e. transfer entropy) clarified directionality to be bottom-up in the default mode network [15]. These findings lead to the hypothesis that when TMS is applied to incoming areas within a relevant brain network, the outgoing areas are modulated; however, the converse is not true. Although our study revealed the possibility that sensory areas could be candidate, incoming areas in WM, outgoing areas were not clarified. This was because we merely showed a phase synchronization expanse from TMS-targeted areas. To address these issues, future studies should identify detailed brain areas and networks using high spatial-resolution measurements.

Note is that the asymmetry in connectivity results could be due to a TMS threshold being higher in PFC than in temporal or parietal cortex. Unfortunately, this study could not identify whether or not the magnitudes of stimulation modulating the PFC and sensory areas are different. Future study should clarify the issues with the same analysis for the EEG data which is recorded during TMS into the frontal and sensory areas in the resting states.

Although our EEG data clearly showed that a bottom-up network is used in WM, our conclusions are limited by a lack of significant differences in our behavioral data. 
Moreover, our conclusions are limited because there remains an alternative explanation for our results using the top-down mechanisms from the frontal areas to the sensory areas. That is, our results merely reflect facilitated bottom-up processing of the cue stimulus, rather than reflecting manipulation-related activities. Previous studies have demonstrated the close relationships between the top-down processing and working memory performance; the diminished top-down modulation in the posterior regions from the prefrontal regions predicted the decrement of the working memory performance [11]. Thus, future studies need to include tasks designed to control for task difficulty, and relevant behavioral data should be compared.

\section{Conclusion}

In summary, we clarified the existence of a bottom-up network in WM based on the observance of increased TMS-induced theta oscillations in the frontal areas during WM tasks. Our approach to determine information flow by manipulating a global phase synchronization would enable to evaluate network directionality of the other cognitive processing.

\section{Acknowledgements}

The research was supported by Grant-in-Aid for Scientific Research on Innovative 
areas (21120005 and 25119512), Grant-in-Aid for Scientific Research (B)

(26282169), JST PRESTO, and Program to Disseminate Tenure Tracking System MEXT.

We would like to thank Yuji Mizuno, Yosuke Ushiku, Yohei Yamada, and Kenji

Kobata for their supports of our data analyses. 


\section{References}

1. Baddeley, AD. Working Memory, Thought and Action. Oxford: Oxford University Press; 2007.

2. Wager TD, Smith EE. Neuroimaging studies of working memory: a meta-analysis. Cogn Affect Behav Neurosci. 2003;3:255-74.

3. Curtis CE, D’Esposito M. Persistent activity in the prefrontal cortex during working memory. Trends Cogn. Sci. 2003; 7 (9): 415-423.

4. Fries P. A mechanism for cognitive dynamics: Neuronal communication through neuronal coherence. Trends Cogn. Sci. 2005; 9: 474-480.

5. Klimesch W, Freunberger R, Sauseng P, Gruber W. A short review of slow phase synchronization and memory: Evidence for control processes in different memory systems? Brain Res. 2008; 1235: 31-44.

6. Fell J, Axmacher N. The role of phase synchronization in memory processes. Nat. Rev. Neurosci. 2011; 12: 105-118.

7. Jensen $\mathrm{O}$, Tesche CD. Frontal theta activity in humans increases with memory load in a working memory task. Eur. J. Neurosci. 2002; 15: 1395-1399. 
8. Sauseng P, Klimesch W, Schabus M, Doppelmayr M. Fronto-parietal EEG coherence in theta and upper alpha reflect central executive functions of working memory. In: International Journal of Psychophysiology. 2005. pp. 97-103.

9. Kawasaki M, Kitajo K, Yamaguchi Y. Dynamic links between theta executive functions and alpha storage buffers in auditory and visual working memory. Eur. J. Neurosci. 2010; 31: 1683-1689.

10. Kawasaki M, Kitajo K, Yamaguchi Y. Fronto-parietal and fronto-temporal theta phase synchronization for visual and auditory-verbal working memory. Front. Psychol. 2014; 5:

11. Zanto T, Rubens M, Thangavel A, Gazzaley, A. Causal role of the prefrontal cortex in top-down modulation of visual processing and working memory. Nature Neuroscience. 2011; 14; 656-661

12. Paus T, Sipila PK, Strafella AP. Synchronization of neuronal activity in the human primary motor cortex by transcranial magnetic stimulation: an EEG study. J. Neurophysiol. 2001; 86: 1983-1990.

13. Rosanova M, Casali A, Bellina V, Resta F, Mariotti M, Massimini M. Natural frequencies of human corticothalamic circuits. J. Neurosci. 2009; 29: 7679_ 7685. 
14. Thut G, Miniussi C. New insights into rhythmic brain activity from TMS-EEG studies. Trends Cogn. Sci. 2009; 13: 182-189.

15. Kawasaki M, Uno Y, Mori J, Kobata K, Kitajo K. Transcranial magnetic stimulation-induced global propagation of transient phase resetting associated with directional information flow. Front. Hum. Neurosci. 2014; 8: 173.

16. Veniero D, Bortoletto M, Miniussi C. TMS-EEG co-registration: On TMS-induced artifact. Clin. Neurophysiol. 2009; 120: 1392-1399.

17. Perrin F, Pernier J, Bertrand O, Echallier JF. Spherical splines for scalp potential and current density mapping. Electroencephalogr. Clin. Neurophysiol. 1989; 72: 184-187.

18. Kayser J, Tenke CE. Principal components analysis of Laplacian waveforms as a generic method for identifying ERP generator patterns: II. Adequacy of low-density estimates. Clin. Neurophysiol. 2006; 117: 369-380.

19. Tallon-Baudry C, Bertrand O, Delpuech C, Pernier J. Stimulus specificity of phase-locked and non-phase-locked $40 \mathrm{~Hz}$ visual responses in human. $J$. Neurosci. 1996; 16: 4240-4249.

20. Lachaux JP, Rodriguez E, Martinerie J, Varela FJ. Measuring phase synchrony in brain signals. Hum. Brain Mapp. 1999; 8: 194-208. 
21. Ilmoniemi RJ, Virtanen J, Ruohonen J, Karhu J, Aronen HJ, Näätänen R, et al. Neuronal responses to magnetic stimulation reveal cortical reactivity and connectivity. Neuroreport 1997; 8: 3537-3540.

22. Massimini M, Ferrarelli F, Huber R, Esser SK, Singh H, Tononi G. Breakdown of cortical effective connectivity during sleep. Science 2005; 309: 2228-2232.

23. Morishima Y, Akaishi R, Yamada Y, Okuda J, Toma K, Sakai K. Task-specific signal transmission from prefrontal cortex in visual selective attention. Nat. Neurosci. 2009; 12: 85-91.

24. Buschman TJ, Miller EK. Top-down versus bottom-up control of attention in the prefrontal and posterior parietal cortices. Science 2007; 315 (5820): 1860-2.

25. Corbetta M, Shulman GL. Control of goal-directed and stimulus-driven attention in the brain. Nat. Rev. Neurosci. 2002; 3 (3): 201-215.

26. Awh E, Jonides J. Overlapping mechanisms of attention and spatial working memory. Trends Cogn. Sci. 2001; 5: 119-126. 
Figure 1. Schematic illustrations of 1 trial sequence for the auditory (A) and visual (V)

working memory (WM) tasks. Yellow hexagons indicated the timing of TMS

applications.

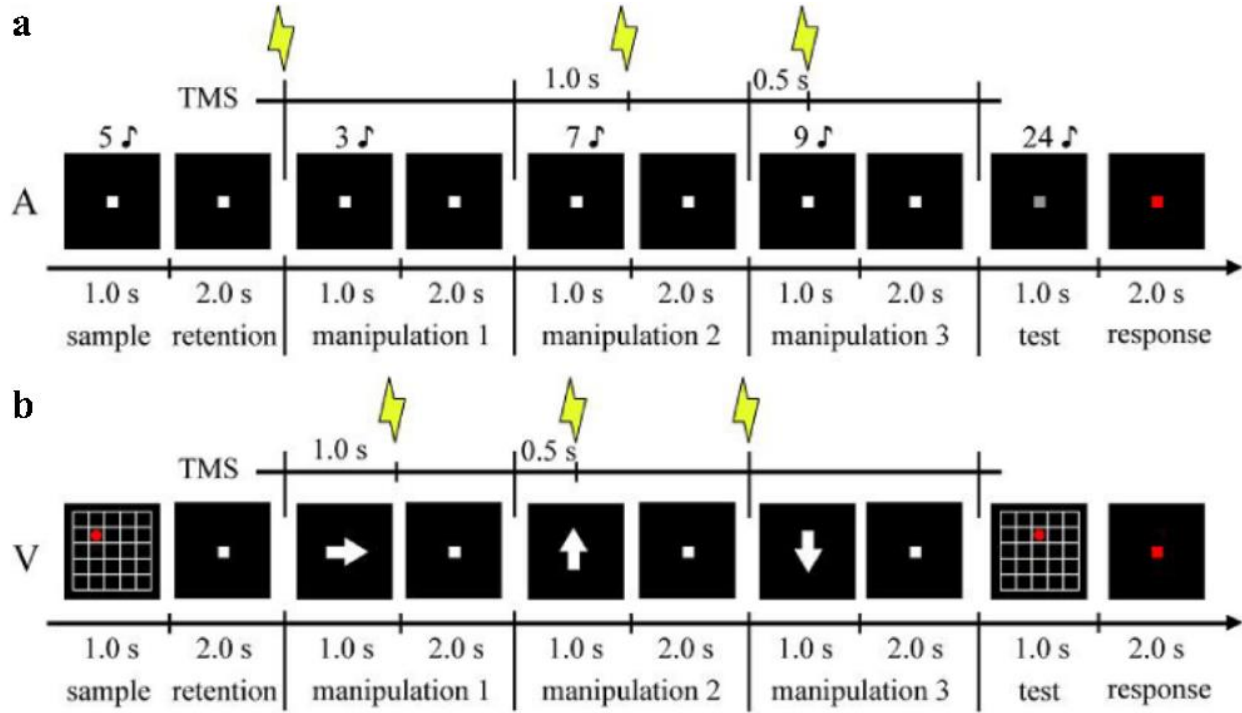


Figure 2. Electrode pairs between the ROI electrodes and the other electrodes showing significantly higher theta (4Hz) PLV for manipulation periods than that for ITI periods ( $p<0.05$; Bonferroni correction). Note that the onset of manipulation phase was 0ms.The significant PLV between the frontal and other electrodes (upper) and between the temporal and other electrodes (lower) under no-TMS (a), frontal-TMS (c), and temporal-TMS (e) conditions during AWM task. The significant PLV between frontal and other electrodes (upper) and between parietal and other electrodes (lower) under no-TMS (b), frontal-TMS (d), and parietal-TMS (f) conditions during VWM task. In figure (c), (d), (e), and (f), TMS was applied at 1000ms. 


\section{Audikny Mis}

(ij) No-'ThIS

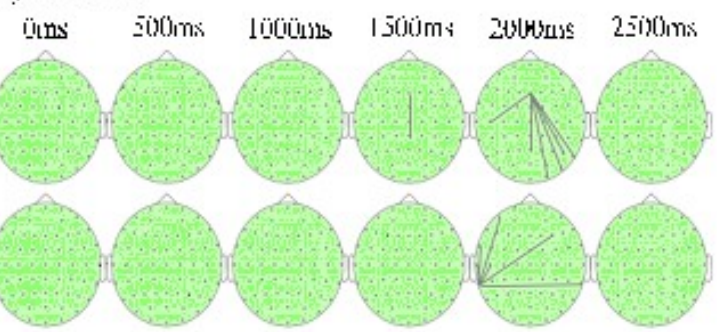

(c) Fromatal-l|asis

4

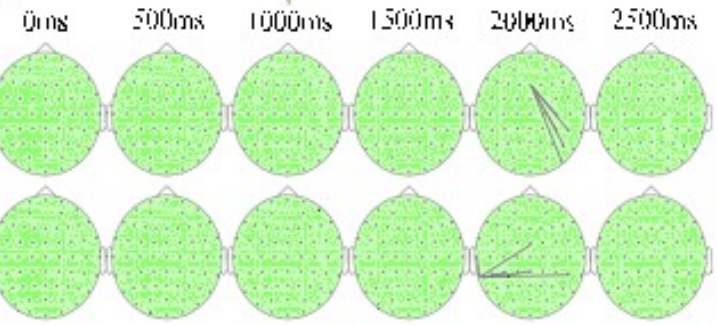

(i) Timpinal-T WS

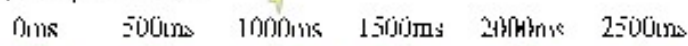

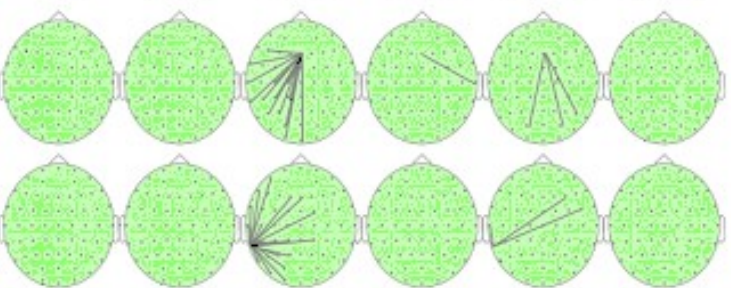

Vis̆La] WM

(b) No-TNSS

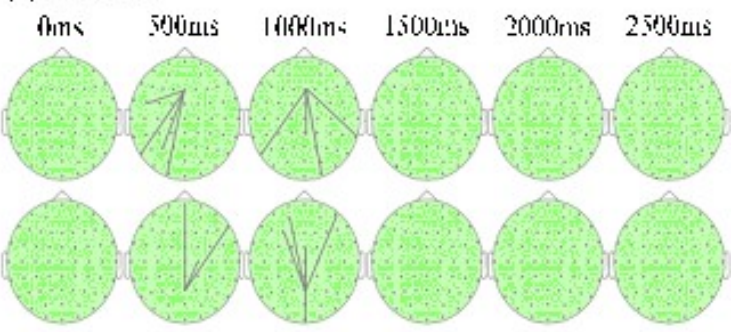

(d) Tron-al-This
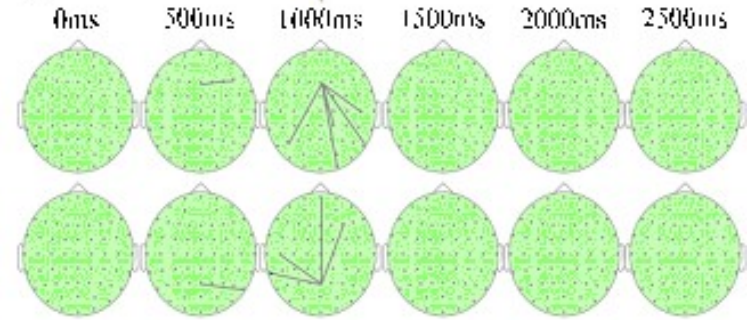

(t) Itariezal-This 4

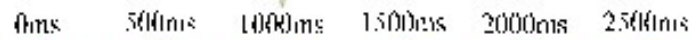

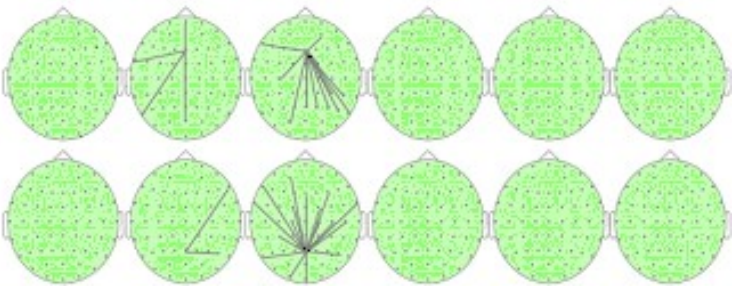




\section{Figure 3.}

The average counted number of electrode pairs showing significantly higher theta $(4 \mathrm{~Hz})$ PLV for manipulation periods than that for ITI periods ( $p<0.05$; Bonferroni correction) among the 6 latencies (0ms, 500ms, 1000ms, 1500ms, 2000ms, and 2500ms) and among the 3 TMS application timings (0ms, 500ms, and 1000ms). Significant pairs between the frontal and the other electrodes (blue), between the temporal and the other electrodes (yellow), and between the temporal and the other electrodes (green) under no-TMS (N), sham-TMS (S), frontal-TMS (F), temporal-TMS (T), and parietal-TMS (P) conditions. The differences between conditions were analyzed by Cui-squared test with Bonferroni-corrected multiple comparison $(*: p<0.05)$.

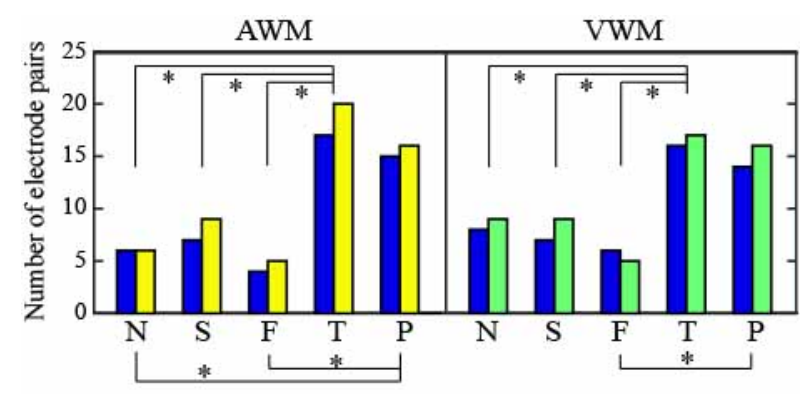

